\title{
Optical spin-orbit interactions in molecular scattering of twisted light
}

\author{
Kayn A. Forbes and David L. Andrews* \\ School of Chemistry, University of East Anglia, Norwich NR4 7TJ, United Kingdom
}

\begin{abstract}
The unique role that can electric quadrupole transition moments play in chiroptical interactions has recently been established with twisted light beams. Manifesting a spin-orbit interaction in paraxial light, the engagement of electric quadrupoles in electronic transitions is highlighted in optical phenomena such as absorption, in both chiral and achiral media. However, spin-orbit interactions of light are also well-known in scattering from small particles, and recent exploratory experimental work suggests a chiroptical interaction of this nature in the scattering of optical vortex beams. Using a quantum electrodynamical formulation, such a sensitivity to the handedness of a vortex beam is accounted for in molecular scattering processes.
\end{abstract}

Keywords: Chirality, scattering, twisted light, optical vortex, nanoparticle, photon interactions, optical activity, orbital angular momentum, spin-orbit coupling, quadrupole, chiroptical effect

\section{INTRODUCTION}

In the wide realm of optics, the scattering of light is one of the most widely studied physical processes. It is responsible for many familiar optical phenomena in solids and at surfaces such as refraction and reflection, as well as the luminosity of the daytime sky. In molecular Rayleigh (elastic) and Raman (inelastic) scattering, the leading contribution to the rate of scattering comes from electric dipole (E1) interactions, and it is accordingly proportional to the frequency-dependent molecular electric polarizability. However, when the molecular matter is chiral, higher-order multipolar couplings can play an important role that leads to novel physics ${ }^{1}$. Specifically, the inclusion of both magnetic dipole (M1) and electric quadrupole (E2) interactions with the incident field leads of discriminatory scattering rates, termed circular differential scattering $(\mathrm{CDS})^{2,3}$. In CDS, chiral molecules scatter left- and right-circularly polarized light at different rates (whereas the leading E1 contribution to scattering is insensitive to the handedness of the incident light). These chiroptical interactions are important for a wide range of spectroscopic techniques, in particular Raman optical activity ${ }^{4,5}$.

Discriminatory interactions between chiral matter and circularly polarized light are the definitive hallmark of a chiroptical interaction. Further examples besides CDS include circular dichroism (CD), optical rotation, and discriminatory forces in optical trapping and binding ${ }^{6}$. All of these rely on an interplay between the handedness of the molecule and light polarization components present within the total light-matter system ${ }^{7}$. The origin of the handedness of molecular matter stems from certain molecules lacking a centre of inversion or plane of symmetry, and thus they differ from their mirror images: as such, they may be denoted either left- or right-handed forms of enantiomeric pairs. For light, the handedness is more commonly associated with spin aspects of the circularly-polarized state: in the optics convention right-handed polarization traces out a helix in a clockwise fashion; left-handed twists anticlockwise as the light propagates towards in in-beam observer. Circularly-polarized light has helicity eigenvalues of \pm 1 , where the positive sign relates to left-handed light, and the negative sign to right-handed, and it therefore delivers a spin angular momentum (SAM) of $\pm \hbar$ per photon on absorption during an electronic transition. 
SAM, however, is not the only form of angular momentum light may possess. The study of light's optical orbital angular momentum (OAM) is now an extremely well-established field, and the rate of new physics it is providing is continually increasing $^{8-12}$. One of the fundamental concepts to have been established is that a beam of light can possess and convey an OAM of $\pm \ell \hbar$ per photon (in the paraxial approximation), where $\ell$ denotes the topological charge (taking on any integer value from 0 to $\infty)^{13}$. The necessary structure for a beam of light to carry this optical OAM is for it to possess a phase factor of $\mathrm{e}^{ \pm i \ell}$, which traces out a helical structure along the beam axis. The most well-studied form of beam that fits this criterion is a Laguerre-Gaussian (LG) mode, whose azimuthal phase imparts a vortex or twisted structure (necessarily associated with an intensity null in the beam centre). This twist is manifested in the OAM per photon by the \pm sign: the light can have either a negative or positive OAM depending, on the direction in which it is twisting.

An obvious question arises: just as the handedness of circularly polarized light produces chiroptical interactions, can the handedness of a twisted beam also produce discriminatory optical processes with matter? An initial answer was first presented nearly two decades ago, quantum electrodynamical theory (QED) being used to conclude that the handedness of a structured light beam should play no role in chiroptical interactions ${ }^{14}$. Provoked by this theoretical work, complementary and supportive experimental observations soon followed, based on experimental conditions assumed by the theory ${ }^{15,16}$. However, in the last few years, some further experimental studies, looking at systems under different conditions, have shown evidence of the contrary - that in certain circumstances the handedness of twisted light can exert a chiroptical influence. These studies, invoking spin-orbit interactions (SOI) ${ }^{17}$ of the incident light, have been able to induce chiroptical effects with OAM by utilizing the helicity-dependent intensity distributions that arise through the SOI of focused nonparaxial vortex beams with circular polarization. Broadening the definition of 'circular dichroism', fundamentally related effects have been identified in non-chiral nanostructures ${ }^{18}$; effects of a similar kind have also been discovered in achiral atomic matter ${ }^{19}$, chiral mesostructures ${ }^{20}$, and by the use of so-called 'spin-orbit beams' to characterize material chirality ${ }^{21}$. Other studies have investigated the exploitation of plasmonic coupling in material interactions with twisted light to engineer chiroptical effects ${ }^{22-28}$, with analogous effects proving to be manifest in vortex electron beams ${ }^{29}$, atomic BoseEinstein condensates ${ }^{30}$ and OAM-induced X-ray dichroism ${ }^{31}$. There are also reports of 'magneto-orbital' dichroism, an OAM analogue of magnetic circular dichroism ${ }^{32}$, and the use of stimulated parametric down conversion to produce a dichroic-like effect through direct action of the OAM in an incident field ${ }^{33}$. Studies looking at Mie scattering ${ }^{34,35}$ also suggest that the sign of $\ell$ in the incident beam is important in these processes, and it has recently emerged that there exists an optical OAM-sensitive transmission rate of LG beams through mouse brain tissue ${ }^{36}$.

Recent QED analyses ${ }^{37,38}$ of single-photon absorption by molecular matter has elucidated an SOI mechanism which delineates how a chiral molecule (and achiral) absorbs a freely-propagating, paraxial Laguerre-Gaussian mode photon with $+\ell$ at a different rate to that of a photon with $-\ell$. In other words, the direction a vortex beam is twisting alters the absorption rate. A number of key general physical principles were discovered in these recent treatments, and these will be utilised in the present analysis that now specifically addresses scattering, rather than absorption. The most important is the role that electric quadrupole transition moments play in chiroptical interactions with a sensitivity to the topological charge of a vortex beam.

\section{SCATTERING THEORY}

To begin our analysis of the scattering of twisted light by chiral molecules we write the interaction Hamiltonian as

$$
\begin{aligned}
H_{\mathrm{int}} & =\sum_{\xi}\left[-\boldsymbol{\mu}(\xi) \cdot \boldsymbol{e}^{\perp}\left(\boldsymbol{R}_{\xi}\right)-Q_{i j}(\xi) \nabla_{j} e_{i}^{\perp}\left(\boldsymbol{R}_{\xi}\right)-\boldsymbol{m}(\xi) \cdot \boldsymbol{b}\left(\boldsymbol{R}_{\xi}\right) \ldots+\text { h.o.t. }\right] \\
& +\frac{e^{2}}{8 m} \sum_{\xi, \alpha}\left[\left(\boldsymbol{q}_{\alpha}(\xi)-\boldsymbol{R}_{\xi}\right) \times \boldsymbol{b}\left(\boldsymbol{R}_{\xi}\right)\right]^{2} \ldots+\text {...h.o.t. },
\end{aligned}
$$

where, for a molecule $\xi$ positioned at $\boldsymbol{R}_{\xi}, \boldsymbol{\mu}$ is the transition electric dipole (E1) moment operator; $\boldsymbol{Q}$ is the transition electric quadrupole (E2) operator and $\boldsymbol{m}$ is the transition magnetic dipole (M1) moment operator: the final term in (1) is 
the leading order diamagnetic interaction term, and $\boldsymbol{q}_{\alpha}(\xi)$ is the position vector of an electron $\alpha$ possessing a charge $e$ with mass $m$. This is the Power-Zienau-Woolley (PZW) quantum interaction Hamiltonian, which couples the molecular transition moments with the fundamental electromagnetic field. Since it does not depend on any gauge potentials, it is inherently gauge-invariant. This, along with the fact all Coulombic interactions are already taken account and causality is accounted for, makes the PZW Hamiltonian the most widely utilised Hamiltonian in modern optical studies ${ }^{39}$. As has previously been established ${ }^{37}$, in the ensuing calculations we are required to include the E2 interactions in our calculations if we wish to observe a sensitivity to the sign of the topological charge in an optical interaction. The same work also highlights that we may also neglect the M1 interactions for our purposes here of finding the leading-order contribution to any potential discriminatory processes dependent on the sign of $\ell$. Although in any multiphoton process the diamagnetic term should be included along with the M1 response to yield gauge-invariant results ${ }^{40}$, in standard two-photon scattering it cannot play any role in chiroptical phenomena for twisted light as it doesn't allow for an E2 interaction. As such, we need only deploy the electric field mode expansion for Laguerre-Gaussian light cast in the paraxial approximation. It emerges as the following function of the cylindrical coordinates: the off-axis radial distance $r$, axial position $z$ and azimuthal angle $\phi:^{41}$

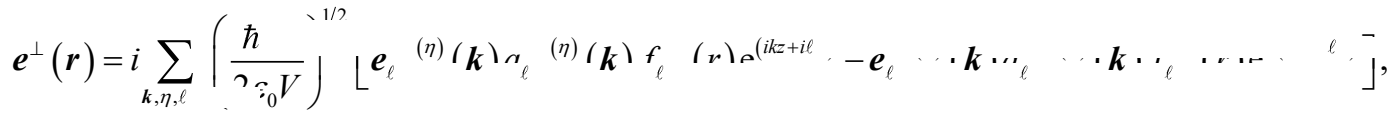

where $a_{\ell}{ }^{(\eta)}(\boldsymbol{k})$ and $a_{\ell}{ }^{\dagger(\eta)}(\boldsymbol{k})$ are the annihilation and creation operators for a photon of mode $\left(\boldsymbol{k}, \eta, \ell,, \boldsymbol{e}_{\ell}{ }^{(\eta)} \boldsymbol{(}_{\boldsymbol{k}}\right)$ is a unit polarization vector transverse to $\boldsymbol{k}$, and $\left.f_{\ell} \quad \ldots\right)$ is a radial distribution function which has no dependence on the sign of $\ell$.

In the following analysis we will restrict ourselves to Rayleigh scattering, with the foresight that the results are then also readily manipulated to account for inelastic Raman scattering. (For Raman scattering, the process engages the derivatives of the various scattering tensors, with respect to the normal coordinates of molecular vibration). At the fundamental level, either form of scattering is a two-photon event, and therefore second-order time-dependent perturbation theory is to be implemented, where the interaction Hamiltonian acts upon a system of $n$ photons in the mode $(k, \eta, \ell$, and molecule $\xi$ in the initial state $\left|n\left(k, \eta, \ell, \quad \boldsymbol{k}^{\prime}, \eta^{\prime}\right) ; E_{0}(\xi)\right\rangle$; the final state of the system is $\left|n-1\left(k, \eta, \ell, \quad \boldsymbol{k}, \eta^{\prime}\right) ; E_{0}(\xi)\right\rangle$, where the scattered photon is in the mode $\left(\boldsymbol{k}^{\prime}, \eta^{\prime}\right)$. To produce the leading order chiroptical interaction with a potential dependence on topological charge, we require the time-order diagrams in Figure 1: these Feynman graphs will produce the E1E2 matrix element.

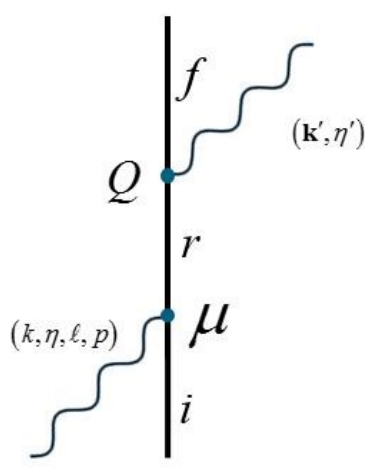

A

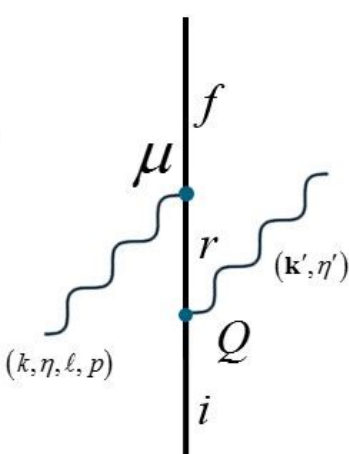

B

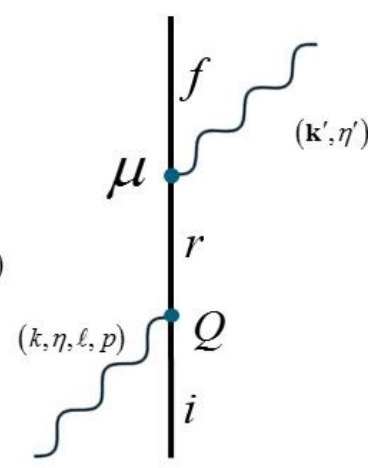

C

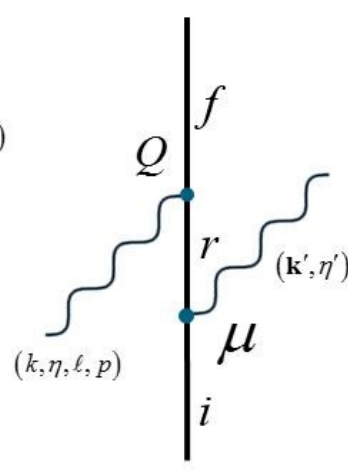

D

Figure 1: The four Feynman time-ordered diagrams that relate to the calculation of the total E1E2 contribution to Rayleigh or Raman scattering of an incident photon of mode $(k, \eta, \ell$, . 
Since the interaction involves quadrupole couplings, to calculate the matrix element requires determination of the gradient of the electric field. This has previously been calculated for Laguerre-Gaussian modes $^{38}$ and is given as follows:

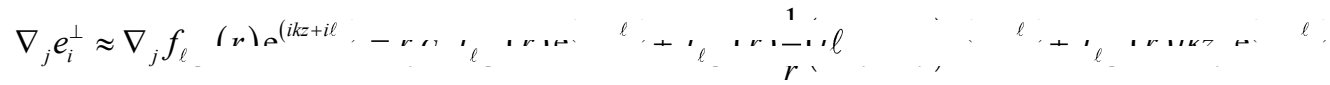

Clearly, for our purposes we are only interested in the first term of the middle term that depends on the sign of $\ell$. Therefore in the ensuing matrix element calculation we neglect the other three terms, concentrating specifically on the $\left.f_{\ell} \quad n\right) \mathrm{e}^{(i k z+i \ell}, \quad \ell$ 'term (though later $\mathrm{n}$ in the analysis we shall include the final term of (3), as this gives the standard CDS result for paraxial light ${ }^{3}$ ). The relevant part of the scattering matrix element now takes the form:

$$
\begin{aligned}
& M_{f i}\left(\ell, \quad\left(\frac{\hbar}{2 \varepsilon_{0} V}\right)^{\vee} v f_{\ell}(n) \overrightarrow{e_{i}} e_{k}\right.
\end{aligned}
$$

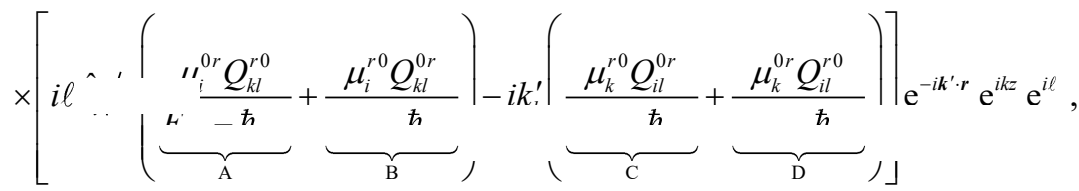

where the A, B, C, and D terms are the corresponding contributions from the Feynman graphs in Figure 1. We now define a frequency-dependent electric dipole-quadrupole polarizability with $k l$-index symmetry as

$$
A_{i k l}(\omega)=\sum_{r}\left(\frac{\mu_{i}^{0 r} Q_{k l}^{r 0}}{E_{r 0}-\hbar}+\frac{Q_{k l}^{0 r} \mu_{i}^{r 0}}{\hbar}\right)
$$

allowing the full $\left(E 1^{2}+\mathrm{E} 1 \mathrm{E} 2\right)$ matrix element to be written succinctly as:

$$
M_{f i}=-\left(\frac{\hbar}{2 \varepsilon_{0} V}\right)^{\vee} n f_{\ell}(r) \bar{e}_{i}^{\prime} e_{k}\left(\alpha_{i k}(\omega)+\frac{i \ell^{\wedge}}{r} A_{i k l}(\omega)-i k_{l}^{\prime} A_{k i l}(\omega)\right) \mathrm{e}^{-i k^{\prime} \cdot r} \mathrm{e}^{i k z} \mathrm{e}^{i \ell} .
$$

In (6) we have now included the dominant E1E1 contribution to the scattering rate, which simply involves the inclusion of the frequency-dependent electric dipole-dipole molecular polarizability tensor $\alpha_{i k}(\omega)$. As we are only interested in the terms that show a linear dependence on $\ell$ (and hence its sign), in the ensuing implementation of the Fermi rule that involves the modulus square of the matrix element we can neglect the E1E1 and E2E2 coupling terms, specifically concentrating on the E1E2 terms to reveal the sought new physics. This is justified on the basis that the E1E1 terms reveal no new physics, whilst the E2E2 terms are typically about $10^{-6}$ smaller than the E1E1 terms, and $10^{-3}$ smaller than the E1E2 terms.

Taking the above into consideration, the rate of E1E2 scattering is calculated using Fermi's Rule:

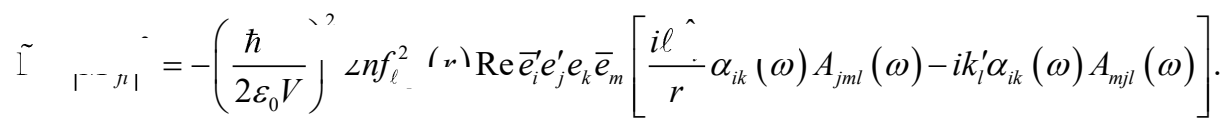

Since expression (7) as it stands includes a factor of the imaginary $i$, to secure a real result and thus an observable scattering rate we need to use either circular or elliptical polarization: the result is null for linearly polarized light. For our purposes here we entertain circularly polarized light, and the following differential rate is secured with the aid of the identity

$$
\begin{aligned}
& e_{k}^{\mathrm{L} / \mathrm{R}} \bar{e}_{m}^{\mathrm{L} / \mathrm{R}}=\frac{1}{2}\left[\left(\delta_{k m}-\hat{k}_{k} \hat{k}_{m}\right) \mp \quad \ldots \ldots . . .\right. \\
& \tilde{\tau^{\prime}} \quad \sim\left(\frac{\hbar}{2 \varepsilon_{0} V}\right)^{2}\left\langle n f_{\ell}^{2}\left({ }_{n}\right) \vec{e}_{i}^{\prime} e_{j}^{\prime} \varepsilon_{k m n} \hat{k}_{n}\left[\frac{\ell^{\wedge}}{r} \alpha_{i k}(\omega) A_{j m l}(\omega)-k^{\prime} \hat{k}_{l} \alpha_{i k}(\omega) A_{m j l}(\omega)\right]\right. \text {, }
\end{aligned}
$$


where $\varepsilon_{k m n}$ is a Levi-Civita totally index-antisymmetic tensor. By isolating the term that only depends on $\ell$, we can explicitly highlight the SOI taking place, identifiable through the following contribution to the scattering rate:

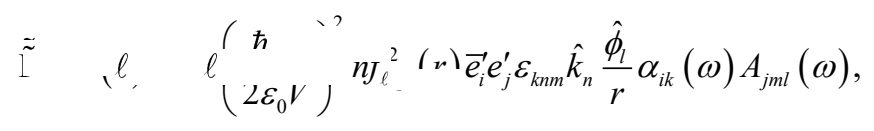

where $\sigma= \pm 1$ represents the helicity eigenvalues for the circular polarization states (SAM) - the positive sign corresponds to left-handed circular polarization, and negative to right-handed. The $\sigma \ell$ factor highlights the SOI of light taking place in mediating the physics underlying this process. A thorough discussion of novel SOI in paraxial light beams and their role in chiroptical processes recently published ${ }^{38}$, affords a context in which to view this new form of manifestation in a scattering interaction.

The rate (8) in its current form corresponds to a molecular system that has positional order, either total or partial. This total (or partial degree) of order has proven to be another key criteria in discriminatory absorption of twisted photons by chiral media. However, it will now be shown that in the scattering of twisted photons by chiral molecules, a chiroptical effect still manifests even in isotropic systems. To secure a result applicable to liquids and gases, we require the total rotational averaging of (8) to yield the scattering rate for an isotropic molecular system. The required spatial averaging of the fifthrank tensor $\alpha_{i k} A_{j m l}$ involves the $I^{(5)}$ scheme $^{42}$ :

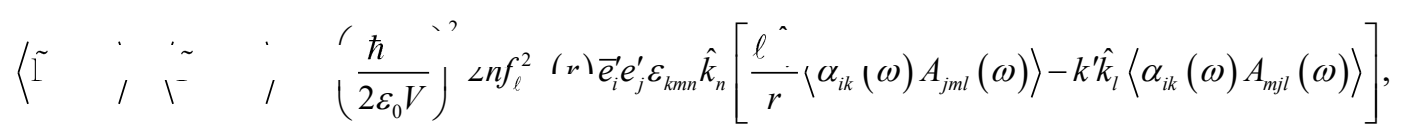

in which angular brackets denote averaging, and Latin and Greek indices refer to space-fixed and molecule-fixed frames, respectively. Carrying out the average, the total rate for $N$ molecules, including the density of final states $\rho_{f}$ from the full Fermi rule, is given as

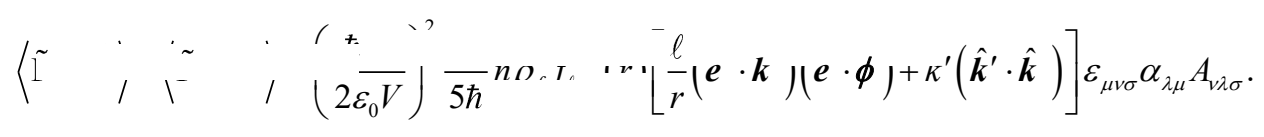

Due to the fact circularly polarized photons are being scattered, we should also include the standard CDS result for paraxial LG beams in order to account for the full E1E2 differential scattering

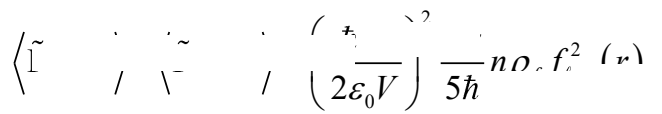

$$
\begin{aligned}
& \times\left[\frac{\ell}{r}\left(\boldsymbol{e} \cdot \boldsymbol{k} \mu \overline{\boldsymbol{e}}^{\prime} \cdot \hat{\boldsymbol{\phi}}\right)+k\left(\boldsymbol{e}^{\prime} \cdot \hat{\boldsymbol{k}}\right)\left(\overline{\boldsymbol{e}}^{\prime} \cdot \hat{\boldsymbol{z}}\right)+k^{\prime}\left(\hat{\boldsymbol{k}}^{\prime} \cdot \hat{\boldsymbol{k}}\right)-\frac{k}{3}\right] \varepsilon_{\mu \nu \sigma} \alpha_{\lambda \mu} A_{\nu \lambda \sigma} .
\end{aligned}
$$

A more useful way of writing the result (12) is in terms of the corresponding difference in scattered radiant intensity for the circularly polarized light:

$$
\Delta I\left(\boldsymbol{k}^{\prime}\right)=-\frac{N I_{0} k^{5}}{240 \varepsilon_{0}^{2} \pi^{2}} f_{\ell}^{2}{ }_{r} r\left[1-3\left(\boldsymbol{e}^{\prime} \cdot \hat{\boldsymbol{k}}\right)\left(\overline{\boldsymbol{e}}^{\prime} \cdot \hat{\boldsymbol{z}}\right)-3\left(\hat{\boldsymbol{k}}^{\prime} \cdot \hat{\boldsymbol{k}}\right)-\frac{3 \ell}{k r}\left(\boldsymbol{e} \cdot \boldsymbol{k} \mu \overline{\boldsymbol{e}}^{\prime} \cdot \hat{\boldsymbol{\phi}}\right)\right] \varepsilon_{\mu \nu \sigma} \alpha_{\lambda \mu} A_{\nu \lambda \sigma}
$$

The first three terms in square brackets in (13) constitute similar physics to the well-known result for circular-differential scattering of plane-wave light ${ }^{3}$, the difference here being that they account for the structure of the incident LaguerreGaussian light. The final term in square brackets, with its linear dependence on $\ell$ (and therefore its sign), represents the new differential scattering interaction. For a given molecular handedness, the scattered radiant intensity is therefore different for an incident LG beam with $+\ell$ (left-handed) as opposed to $-\ell$ (right-handed) twist. Equally, for fixed $\ell$, changing a chiral molecule to its corresponding enantiomer will also change the sign of the difference of scattered intensity 
through $\alpha_{\lambda \mu} A_{v \lambda \sigma}$ changing sign. At this stage we note that for the complete result, E1M1 interactions may also be included, as we shall demonstrate in subsequent work. However, since the electric quadrupole terms are specifically sensitive to the phase gradient of the incident electromagnetic radiation, the result as presented here should represent the leading result for high-value topological charge.

\section{SCATTERING GEOMETRY}

The equation for scattered radiant intensity can be further manipulated for experimental application by introducing a scattering geometry as shown in Figure 2. The polarization of the scattered radiation can be resolved by looking at two orthogonal polarizations - one in the plane of the scattered wave vector and the incident polarization, and the other perpendicular to the plane.

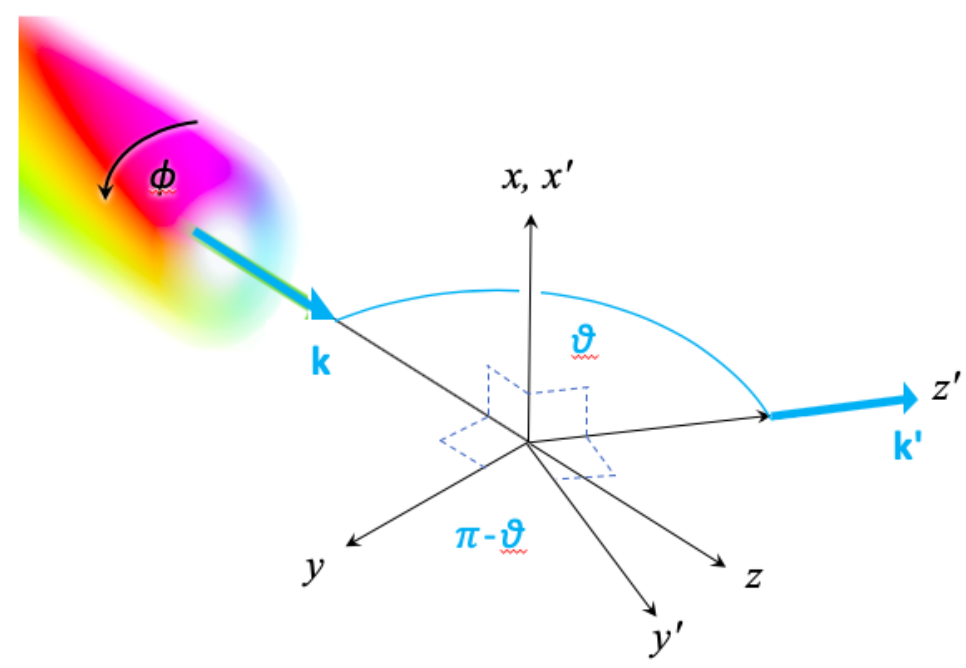

Figure 2: Scattering geometry for a chiral molecule situated at the origin.

To proceed we make use of the following coordinate transformation $\hat{\phi}=-\hat{x} \sin \phi+\hat{y} \cos \phi$, which allows the scattered radiant intensity to be written as;

$$
\begin{aligned}
& \Delta I\left(\boldsymbol{k}^{\prime}\right)=-\frac{N I_{0} k^{5}}{240 \varepsilon_{0}^{2} \pi^{2}} f_{\ell}^{2}(r)\left[1-3\left(\boldsymbol{e}^{\prime} \cdot \hat{\boldsymbol{k}}\right)\left(\overline{\boldsymbol{e}}^{\prime} \cdot \hat{\boldsymbol{z}}\right)-3\left(\hat{\boldsymbol{k}}^{\prime} \cdot \hat{\boldsymbol{k}}\right)-\frac{3 \ell}{k r}\left(\boldsymbol{e} \cdot \boldsymbol{k} \mu \overline{\boldsymbol{e}}^{\prime} \cdot(-\hat{\boldsymbol{x}} \sin \phi+\hat{\boldsymbol{y}} \cos \phi)\right)\right] \varepsilon_{\mu \nu \sigma} \alpha_{\lambda \mu} A_{\nu \lambda \sigma} \\
& \therefore \Delta I\left(\boldsymbol{k}^{\prime}\right)=-\frac{N I_{0} k^{\prime 4}}{240 \varepsilon_{0}^{2} \pi^{2}} f_{\ell}^{2}\left({ }_{r}\right)\left[k-3 k\left(\boldsymbol{e}^{\prime} \cdot \hat{\boldsymbol{k}}\right)\left(\overline{\boldsymbol{e}}^{\prime} \cdot \hat{\boldsymbol{z}}\right)-3 k^{\prime}\left(\hat{\boldsymbol{k}}^{\prime} \cdot \hat{\boldsymbol{k}}\right)-\frac{3 \ell}{r} \sin \sigma \cos \theta \cos \phi\right] \varepsilon_{\mu \nu \sigma} \alpha_{\lambda \mu} A_{\nu \lambda \sigma} .
\end{aligned}
$$

Evaluating the result for two orthogonal exit polarizations and summing the result thus leads to the following expression as the total E1E2 contribution to circular differential scattering;

$$
\left.\tilde{r}_{1},=\mp \ell_{\left(2 \varepsilon_{0} V\right.}^{(\hbar}\right)^{\prime} \rho_{f} \frac{2 \pi}{\hbar} \frac{n}{2} f^{2}(r) \sin 2 \theta \cos \phi \varepsilon_{\mu \sigma \pi} \alpha_{\lambda \mu} A_{\sigma \pi \lambda},
$$

which vanishes for $\theta=0, \pi / 2$ etc. Interestingly, the spatial distribution of the scattering associated with this mechanism therefore exhibits the intrinsic symmetry of a quadrupole. 


\section{DISCUSSION}

The significance of E2 quadrupole interactions between molecular matter and twisted light has again been highlighted, specifically by an ability to engender a novel form of scattering optical activity. For chiral molecules irradiated with circularly-polarized LG beams, a portion of the scattered radiant intensity is sensitive to the direction the azimuthal phase front is twisting, manifest through a linear-dependence on the topological charge $\ell$. Although in the case of single-photon absorption, the E1E2 CVD effect was shown to disappear for randomly orientated isotropic molecular systems ${ }^{37}$, in the case of E1E2 chiroptical scattering of vortex light, it has been shown that this is not the case. This mirrors the well-known fact that $A_{i(j k)}$ only contributes to CD in oriented media, but it nonetheless contributes to natural Rayleigh and Raman optical activity in both oriented and isotropic media ${ }^{1,3}$. In common with the CVD effect, the chiroptical scattering phenomena described in this paper are also manifestations of a SOI of light, requiring the vortex light beam to also be circularly (or at least elliptically) polarized. This work represents a theoretical study concerned with scattering optical activity by chiral molecules that provides a background mechanism to interpret recent preliminary experimental studies ${ }^{43,44}$.

\section{REFERENCES}

1. Barron, L. D. Molecular Light Scattering and Optical Activity. (Cambridge University Press, Cambridge, 2009).

2. Barron, L. D. \& Buckingham, A. D. Rayleigh and Raman scattering from optically active molecules. Mol. Phys. 20, 1111-1119 (1971).

3. Craig, D. P. \& Thirunamachandran, T. Molecular Quantum Electrodynamics: An Introduction to RadiationMolecule Interactions. (Dover, Mineola NT, 1998).

4. Barron, L. D., Mutter, S. T. \& Blanch, E. W. Raman optical activity, in Chiral Analysis: Advances in Spectroscopy, Chromatography and Emerging Methods ed. Polavarapu, P. L., 249-291 (Elsevier, 2018).

5. Barron, L. D., Hecht, L., McColl, I. H. \& Blanch, E. W. Raman optical activity comes of age. Mol. Phys. 102, 731744 (2004).

6. Bradshaw, D. S., Forbes, K. A., Leeder, J. M. \& Andrews, D. L. Chirality in optical trapping and optical binding. Photonics 2, 483-497 (2015).

7. Andrews, D. L. Quantum formulation for nanoscale optical and material chirality: symmetry issues, space and time parity, and observables. J. Opt. 20, 033003 (2018).

8. Barnett, S. M., Babiker, M. \& Padgett, M. J. Optical Orbital Angular Momentum. (The Royal Society, 2017).

9. Rubinsztein-Dunlop, H. et al. Roadmap on structured light. J. Opt. 19, 013001 (2016).

10. Andrews, D. L. Structured Light and its Applications: An Introduction to Phase-Structured Beams and Nanoscale Optical Forces. (Academic Press, New York, 2011).

11. Andrews, D. L. \& Babiker, M. The Angular Momentum of Light. (Cambridge University Press, 2012).

12. Babiker, M., Andrews, D. L. \& Lembessis, V. E. Atoms in complex twisted light. J. Opt. 21, 013001 (2019). 
13. Allen, L., Beijersbergen, M. W., Spreeuw, R. J. C. \& Woerdman, J. P. Orbital angular momentum of light and the transformation of Laguerre-Gaussian laser modes. Phys. Rev. A 45, 8185 (1992).

14. Andrews, D. L., Romero, L. D. \& Babiker, M. On optical vortex interactions with chiral matter. Opt. Commun. 237, 133-139 (2004).

15. Araoka, F., Verbiest, T., Clays, K. \& Persoons, A. Interactions of twisted light with chiral molecules: An experimental investigation. Phys. Rev. A 71, 055401 (2005).

16. Löffler, W., Broer, D. J. \& Woerdman, J. P. Circular dichroism of cholesteric polymers and the orbital angular momentum of light. Phys. Rev. A 83, 065801 (2011).

17. Bliokh, K. Y., Rodríguez-Fortuño, F. J., Nori, F. \& Zayats, A. V. Spin-orbit interactions of light. Nat. Photonics $\mathbf{9}$, 796 (2015).

18. Zambrana-Puyalto, X., Vidal, X. \& Molina-Terriza, G. Angular momentum-induced circular dichroism in nonchiral nanostructures. Nat. Commun. 5, 4922 (2014).

19. Afanasev, A., Carlson, C. E. \& Solyanik, M. Circular dichroism of twisted photons in non-chiral atomic matter. $J$. Opt. 19, 105401 (2017).

20. Ni, J. et al. Ultra-sensitive experimental detection of chiral meso-structures by orbital angular momentum of light. ArXiv Prepr. ArXiv180902754 (2018).

21. Samlan, C. T., Suna, R. R., Naik, D. N. \& Viswanathan, N. K. Spin-orbit beams for optical chirality measurement. Appl. Phys. Lett. 112, 031101 (2018).

22. Brullot, W., Vanbel, M. K., Swusten, T. \& Verbiest, T. Resolving enantiomers using the optical angular momentum of twisted light. Sci. Adv. 2, e1501349 (2016).

23. Wu, T., Wang, R. \& Zhang, X. Plasmon-induced strong interaction between chiral molecules and orbital angular momentum of light. Sci. Rep. 5, 18003 (2015).

24. Wang, S. et al. Angular Momentum-dependent transmission of circularly polarized vortex beams through a plasmonic coaxial nanoring. IEEE Photonics J. 10, 1-9 (2018).

25. Ren, H. \& Gu, M. Angular momentum-reversible near-unity bisignate circular dichroism. Laser Photonics Rev. 12, 1700255 (2018).

26. Reddy, I. V., Baev, A., Furlani, E. P., Prasad, P. N. \& Haus, J. W. Interaction of structured light with a chiral plasmonic metasurface: Giant enhancement of chiro-optic response. ACS Photonics 5, 734-740 (2018).

27. Kerber, R. M. et al. Interaction of an Archimedean spiral structure with orbital angular momentum light. New $J$. Phys. 20, 095005 (2018).

28. Kerber, R. M., Fitzgerald, J. M., Oh, S. S., Reiter, D. E. \& Hess, O. Orbital angular momentum dichroism in nanoantennas. Commun. Phys. 1, 87 (2018). 
29. Asenjo-Garcia, A. \& de Abajo, F. G. Dichroism in the interaction between vortex electron beams, plasmons, and molecules. Phys. Rev. Lett. 113, 066102 (2014).

30. Mondal, P. K., Deb, B. \& Majumder, S. Optical manipulation of matter-wave vortices: An analog of circular dichroism. Phys. Rev. A 92, 043603 (2015).

31. van Veenendaal, M. \& McNulty, I. Prediction of strong dichroism induced by X rays carrying orbital momentum. Phys. Rev. Lett. 98, 157401 (2007).

32. Mathevet, R., de Lesegno, B. V., Pruvost, L. \& Rikken, G. L. Negative experimental evidence for magneto-orbital dichroism. Opt. Express 21, 3941-3945 (2013).

33. Lowney, J., Roger, T., Faccio, D. \& Wright, E. M. Dichroism for orbital angular momentum using parametric amplification. Phys. Rev. A 90, 053828 (2014).

34. Garbin, V. et al. Mie scattering distinguishes the topological charge of an optical vortex: a homage to Gustav Mie. New J. Phys. 11, 013046 (2009).

35. Zambrana-Puyalto, X., Vidal, X., Woźniak, P., Banzer, P. \& Molina-Terriza, G. Tailoring multipolar Mie scattering with helicity and angular momentum. ACS Photonics 5, 2946-2944 (2018).

36. Mamani, S. et al. Transmission of classically entangled beams through mouse brain tissue. J. Biophotonics e201800096 (2018).

37. Forbes, K. A. \& Andrews, D. L. Optical orbital angular momentum: twisted light and chirality. Opt. Lett. 43, 435438 (2018).

38. Forbes, K. A. \& Andrews, D. L. Spin-orbit interactions and chiroptical effects engaging orbital angular momentum of twisted light in chiral and achiral media. ArXviv Prepr. ArXiv180905470 (2018).

39. Andrews, D. L., Jones, G. A., Salam, A. \& Woolley, R. G. Perspective: Quantum Hamiltonians for optical interactions. J. Chem. Phys. 148, 040901 (2018).

40. Forbes, K. A. Role of magnetic and diamagnetic interactions in molecular optics and scattering. Phys. Rev. A 97, 053832 (2018).

41. Romero, L. D., Andrews, D. L. \& Babiker, M. A quantum electrodynamics framework for the nonlinear optics of twisted beams. J. Opt. B Quantum Semiclassical Opt. 4, S66-S72 (2002).

42. Andrews, D. L. \& Thirunamachandran, T. On three-dimensional rotational averages. J. Chem. Phys. 67, 5026-5033 (1977).

43. Milione, G., Secor, J., Michel, G., Evans, S. \& Alfano, R. R. Raman optical activity by light with spin and orbital angular momentum. in Complex Light and Optical Forces V Proc SPIE 7950, 79500H (2011).

44. Bendau, E., Zhang, L., Gozali, R., Ashrafi, S. \& Alfano, R. R. Vortex beams and optical activity of sucrose. in Complex Light and Optical Forces XI, Proc SPIE 10120, 1012004 (2017). 